ARTÍCULO ORIGINAL

\title{
Simulación del secado en madera de Eucalyptus nitens usando modelos de difusión bajo condiciones unidimensionales e isotérmicas
}

\author{
Simulation of drying process in Eucalyptus nitens sawn-wood using the diffusive \\ model in a one-dimensional and isothermal conditions
}

Claudio Montero ${ }^{1}$ (D), Carlos Rozas ${ }^{1}$ (D), Carlos Picarte ${ }^{2}$

${ }^{1}$ Departamento de Ingeniería en Maderas, Universidad del Bío-Bío, Concepción, Chile

${ }^{2}$ Departamento de Matemática, Universidad del Bío-Bío, Concepción, Chile

Como citá: Montero, C., Rozas, C., \& Picarte, C. (2020). Simulación del secado en madera de Eucalyptus nitens usando modelos de difusión bajo condiciones unidimensionales e isotérmicas. Scientia Forestalis, 48(126), e2957. https://doi.org/10.18671/scifor.v48n126.09

\begin{abstract}
Resumen
La simulación es usada ampliamente para mejorar y optimizar el proceso de secado de la madera. De estos, los modelos de difusión se utilizan para predecir el secado en latifoliadas de secado lento y de baja permeabilidad, haciéndolos adecuados para usarse en Eucalyptus nitens. El objetivo de esta investigación, fue simular el gradiente de humedad y la curva de secado en madera tangencial de Eucalyptus nitens. Se evaluaron dos condiciones de secado isotérmica $\left(40 / 35^{\circ} \mathrm{C}\right.$ y $\left.55 / 50{ }^{\circ} \mathrm{C}\right)$ a una velocidad del aire $(2 \mathrm{~m} / \mathrm{s})$, obteniendo el gradiente y curva de secado experimental, siendo comparados con valores entregados por la simulación numérica del modelo. Los resultados mostraron un coeficiente de difusión entre $1,5-4,5 \times 10^{-10}\left(\mathrm{~m}^{2} / \mathrm{s}\right)$, presentando una relación inversa con el contenido de humedad y directa con la temperatura de secado. La simulación mostró un correcto ajuste del gradiente de humedad y curva de secado, incrementando su precisión en las últimas etapas del secado. En vista de lo anterior, el modelo se consideró adecuado y de bajo costo computacional, para predecir el secado en madera aserrada de Eucalyptus nitens.
\end{abstract}

Palabras clave: Coeficiente de difusión efectivo; Modelación matemática; Gradiente de humedad; Curva de secado.

\begin{abstract}
Simulation has been widely used to improve and optimize the wood drying process. Of these, the diffusive models are used to predict the slow-drying hardwood and low permeability species, so it could be suitable to simulate the drying process in Eucalyptus nitens sawn-wood. The aim of this research, was to simulate the moisture gradient and drying curve, in Eucalyptus nitens tangential sawn-wood. Two isothermal drying conditions were evaluated $\left(40 / 35^{\circ} \mathrm{C}\right.$ and $\left.55 / 50^{\circ} \mathrm{C}\right)$ at air velocity of $2 \mathrm{~m} / \mathrm{s}$, and the experimental values of moisture gradient and drying curve were obtained, being compared with values provided by the numerical solution of the model. The results showed a diffusion coefficient between $1.5-4.5 \times 10^{-10}\left(\mathrm{~m}^{2} / \mathrm{s}\right)$, showing an inverse relationship with the moisture content and a direct relationship with the drying temperature. The simulation showed a correct adjustment of the moisture gradient and drying curve, increasing the precision in the last drying stages. Therefore, the model was considered adequate and low computational cost, to predict the drying in Eucalyptus nitens sawn-wood.
\end{abstract}

Keywords: Effective diffusion coefficient; Mathematical modeling; Moisture gradient; Drying curve.

Fuente de financiamiento: Ninguna.

Conflicto de interés: nada que declarar.

Autor correspondiente: cmontero@ubiobio.cl

Recibido: 13 julio 2018

Aceptado: 5 julio 2019

Editor: Francides Gomes da Silva Júnior.

(c) Este es un artículo publicado en acceso abierto (Open Access) bajo la licencia Creative Commons Attribution, que permite su uso, distribución y ceproducción en cualquier medio, sin restricciones siempre que el trabajo original sea debidamente citado. 


\section{INTRODUCCIÓN}

El interés de la industria maderera por mejorar y optimizar sus procesos, ha hecho que la modelación y simulación del secado, se conviertan en herramientas ampliamente utilizadas (Bramhall, 1979; Resch et al., 1989). De este modo, la simulación permite obtener información útil para la toma de decisiones (Liu, 1989; Baronas et al., 2002), evitando el uso de costosos métodos empíricos y experimentales (Rozas et al., 2009).

Para simular el secado de la madera, se deben considerar al transporte del agua libre presente sus cavidades celulares, la difusión del agua higroscópica adherida a las paredes celulares y la difusión del vapor de agua que circula por las células de la madera. A pesar de que estos mecanismos de transporte, son descritos por diferentes principios, la literatura indica que el proceso de secado se puede modelar en su totalidad, como un proceso de difusión que obedece a la ley de Fick (Nadler et al., 1985; Liu, 1989; Chen et al., 1996; Eriksson et al., 2007; Rozas et al., 2009).

Para Simpson (1993), Pang (1996), Olek \& Weres (2007) y Kang et al. (2009), el modelo difusivo permite predecir con alta precisión, el gradiente de humedad y la curva de secado en la madera. Para esto, se debe resolver numéricamente el término de segundo orden del modelo y contar con los coeficientes de difusión de la madera durante el secado. Una vez logrado lo anterior, es posible simular el gradiente de humedad y la curva de secado en la madera (Hart, 1964; Nadler et al., 1985; Simpson, 1993; Rozas et al., 2009).

En un principio, la aplicación del modelo difusivo se limitaba a maderas con humedad, bajo el punto de saturación de las fibras (PSF) (Bui et al., 1980; Nadler et al., 1985; Simpson, 1993; Eriksson et al., 2007). Sin embargo, en los últimos años el modelo se aplicó para simular el secado de maderas sobre el rango higroscópico, mediante la obtención de un coeficiente de difusión efectivo, el cual envuelve la difusión del vapor de agua y del agua higroscópica, así como el flujo de agua capilar, el cual ha presentando resultados con adecuada precisión (Simpson \& Liu, 1997; Hukka, 1999; Rozas, 2007; Rozas et al., 2009; Gatica et al., 2011).

Esta variante en la aplicación del modelo difusivo, hace factible implementarlo en maderas de secado lento y de baja permeabilidad (Nadler et al., 1985; Eriksson et al., 2007). Haciendolo apropiado, para simular el secado en diversas especies de Eucalyptus y latifoliadas de permeabilidad baja o moderada. Ya que, estas especies presentan un flujo restringido del agua capilar, el cual es de similar magnitud al flujo del agua higroscópica (Nadler et al., 1985; Blakemore \& Langrish, 2008).

De igual modo, la aplicación del modelo difusivo para el secado unidimensional de la madera, fue reportado por Pang (1996), con aceptables resultados, quien además reportó un flujo de humedad de las caras de la madera 20 veces mayor al espesor y 100 veces mayor que el ancho. Otra interesante ventaja del modelo difusivo, es que requiere de pocos parámetros para su implementación (Nadler et al., 1985; Simpson, 1993; Chen et al., 1996; Liu \& Simpson, 1999; Cai, 2005; Eriksson et al., 2007). Específicamente, la relación entre el coeficiente de difusión y el gradiente de humedad, permite obtener el flujo de humedad (Hart \& Darwin, 1971), por lo que se debe contar con coeficientes de difusión precisos, para asegurar una simulación satisfactoria (Avramidis \& Siau, 1987: Liu, 1989; Li et al., 2005; Mukam \& Wanko, 2005). En la misma línea, Bui et al. (1980), Chen et al. (1996), Liu et al. (2001), Olek \& Weres (2007), Kang et al. (2009), y Gatica et al. (2011), incrementaron la precisión de la modelación, aplicando métodos numéricos e inversos mediante uso de software, o bien mediante métodos de optimización de la curva de secado (Blakemore \& Langrish, 2008).

Ante estas evidencias, el modelo difusivo muestra ser una herramienta interesante, para predecir la curva de secado y el gradiente de humedad en madera de Eucalyptus nitens. Comprender el secado de esta especie, es relevante para incrementar su utilización, debido a que presenta limitaciones tecnologicas para su uso, tales como pronunciados gradientes de humedad, los que generan colapso severo, asi como tensiones de secado que producen alabeos y deformaciones en la madera. Entonces, los resultados entregados por la simulación, pueden ayudar a la comprensión y conocimiento tecnológico de esta especie, permitiendo buscar estrategias para incrementar la calidad y rendimiento del proceso de secado. Lo anterior, puede impactar positivamente en la cadena de valor y competitividad de la madera de Eucalyptus nitens frente a otras especies. En este sentido, el objetivo de esta investigación, fue predecir matemáticamente a los coeficientes de difusión y simular el gradiente de humedad y curva de secado, durante un proceso de secado isotérmico en madera aserrada de Eucalyptus nitens, empleando el modelo difusivo basado en la segunda ley de Fick, para un rango de humedad de 
la madera comprendido entre la humedad máxima después del aserrío y la humedad de equilibrio para las condiciones de secado preestablecidas.

\section{MATERIALES Y MÉTODOS}

\section{Procedencia de la madera.}

Se utilizaron 10 árboles de Eucalyptus nitens de 12 años, provenientes de la provincia del Biobío, la que presenta clima mediterráneo del tipo Csb de estaciones marcadas de periodos secos y lluviosos similares. La precipitación total es de 1.070 mm/año. El verano es seco y presenta un clima de transición entre marzo y abril, mientras que el invierno presenta precipitaciones en forma de lluvia, bajas temperaturas y frecuentes heladas (Dirección Meteorológica de Chile, 2001).

\section{Proceso de aserrío de trozas.}

Los árboles fueron trozados a 2,0 m de largo y luego trasladados al Pabellón Tecnológico de la Madera (PTM) de la Universidad del Bío-Bío. Para el aserrío, se utilizó un carro huincha con sierra vertical, el cual aplicó un plan de corte que priorizó la obtención de piezas en corte tangencial de $36 \mathrm{~mm}$ de espesor, $100 \mathrm{~mm}$ de ancho y $1.000 \mathrm{~mm}$ de largo.

\section{Preparación de las piezas de control para los ensayos de secado.}

Se seleccionaron 2 piezas de control, para cada condición de secado evaluada. Cada pieza de control, fue sellada con silicona y papel de aluminio en el espesor y ancho. De esta forma, se aseguró un flujo de humedad unidimensional que, en este caso correspondió a las caras de la pieza.

\section{Preparación de las cargas de secado.}

El secado se realizó en una cámara convencional semi-industrial, con capacidad de $0,78 \mathrm{~m}^{3}$, que contaba con una velocidad del aire de hasta $4 \mathrm{~m} / \mathrm{s}$, un sistema de humidificación por vapor y de calefacción por resistencias eléctricas. Para el control de la temperatura de bulbo seco/húmedo, se emplearon termocuplas tipo-J, cuyas lecturas fueron almacenadas en un sistema de adquisición de datos (Data-Logger).

\section{Condiciones de los ensayos de secado.}

Las condiciones de secado evaluadas, se detallan en la Tabla 1. Se utilizaron 2 piezas de control para cada condición de secado, siendo una para controlar la humedad promedio de la pieza y otra para determinar el gradiente de humedad. La cámara de secado fue completada con otras 120 piezas, incluyendo las piezas de control, permitiendo de esta forma simular las condiciones de secado industrial.

Tabla 1. Parámetros de secado utilizados para cada condición evaluada.

\begin{tabular}{lccc}
\hline \multicolumn{1}{c}{ Parámetro de secado } & Unidad & Condición 1 & Condición 2 \\
\hline Temperatura bulbo seco $\left(\mathrm{T}_{\mathrm{bs}}\right)$ & ${ }^{\circ} \mathrm{C}$ & 40 & 55 \\
Temperatura bulbo húmedo $\left(\mathrm{T}_{\mathrm{bh}}\right)$ & ${ }^{\circ} \mathrm{C}$ & 35 & 50 \\
Velocidad del aire $(\mathrm{V})$ & $\mathrm{m} / \mathrm{s}$ & 2 & 2 \\
Humedad relativa $(\mathrm{HR})$ & $\%$ & 76 & 76 \\
Contenido de humedad de equilibrio $(\mathrm{CHE})$ & $\%$ & 13 & 13 \\
Muestras por ensayo & $\mathrm{n}^{\circ}$ & 2 & 2 \\
Réplicas por ensayo & $\mathrm{n}^{\circ}$ & 2 & 2 \\
\hline
\end{tabular}




\section{Obtención experimental del gradiente de humedad.}

El gradiente de humedad experimental, se obtuvo de un cilindro de diámetro $20 \mathrm{~mm}$, extraido con una sierra copa al espesor de la pieza, el cual fue seccionado en una ingletadora en siete láminas de $2,5 \mathrm{~mm}$ de espesor, asumiendo que la mitad superior e inferior, presentaron identica distribucion de humedad. A cada lámina obtenida, se le determinó el contenido de humedad según la norma NCh 176/10f 84 (Instituto Nacional de Normalizacion, 1984). El agujero resultante de la extracción, fue sellado con silicona y cubierto con papel aluminio. Para finalmente, reingresar la pieza en la cámara de secado. Esta operación, fue realizada en intervalos regulares de 12 horas para cada condición de secado evaluada.

\section{Obtención experimental del contenido de humedad.}

El contenido de humedad fue controlado antes, durante y después de cada ensayo. Para esto, se controló la masa de cada pieza de control con una balanza de precisión 0,01 (g), en un tiempo menor a 1 minuto. Esto último, para minimizar el efecto ambiental sobre la pieza de control. Las mediciones se realizaron cada 12 horas, hasta que la pieza de control no tuvo variación de masa (humedad de equilibrio). Después, la pieza de control se secó en estufa a $103{ }^{\circ} \mathrm{C}$ por 24 horas, hasta estado anhidro y mediante la norma NCh 176/10f 84 (Instituto Nacional de Normalizacion, 1984), se obtuvo el contenido de humedad para cada instante de tiempo evaluado.

\section{Desarrollo del modelo difusivo.}

Al aplicar el modelo difusivo, se consideró un flujo de humedad en estado transitorio, con transporte de humedad desde el interior a la superficie de la pieza, siguiendo el comportamiento descrito por la segunda ley de Fick. En este sentido, el modelo no buscó explicar el flujo de los distintos tipos de humedad presentes en la madera. Se utilizó como potencial de flujo, al contenido de humedad, asumiéndolo perpendicular a la superficie y no se consideró la variación dimensional de la madera. En este sentido, el flujo de humedad unidimensional durante el secado de madera de Eucalyptus nitens, viene dado por la Ecuación 1.

$$
\frac{d C H}{d t}=\frac{\partial}{\partial x}\left(D_{e f} \frac{\partial C H}{\partial x}\right)
$$

Dónde; $\mathrm{CH}$ : contenido de humedad $\left(\mathrm{kg}_{\mathrm{ggua}} / \mathrm{kg}_{\text {masa seca) }}\right.$; $\mathrm{x}$ : Coordenada espacial (espesor de la pieza perpendicular al flujo de humedad) (m); t: tiempo (s); $D_{\text {ef: }}$ Coeficiente de difusión efectivo $\left(\mathrm{m}^{2} / \mathrm{s}\right)$.

Para resolver el modelo, primero se definieron las constantes de integración, por lo que se calcularon las condiciones iniciales y de contorno. La primera condición (Ecuación 2), requirió del contenido de humedad inicial (contenido de humedad máximo). La segunda condición de contorno, se relacionó con el flujo convectivo de humedad (Ecuación 3), por lo que requirió del coeficiente convectivo de masa $\left(\mathrm{k}_{\mathrm{m}}\right)$, el cual fue obtenido por las correlaciones empíricas disponibles en la literatura.

$$
\begin{aligned}
& t=0 \rightarrow M=M_{i} \\
& x=0 \rightarrow D \frac{\partial M}{\partial x}=k_{m}\left(M-M_{E}\right) \\
& x=\frac{L}{2} \rightarrow \frac{\partial M}{\partial x}=0
\end{aligned}
$$


La tercera condición (Ecuación 4), también fue de contorno y asumió un flujo nulo al centro de la pieza (condición de simetría). Cabe mencionar que las condiciones de contorno, requirieron del coeficiente de difusión (D) para describir el flujo interno de humedad y del coeficiente convectivo de masa $\left(\mathrm{k}_{\mathrm{m}}\right)$ para describir la evaporación superficial de la pieza durante el secado.

El término de segundo orden del modelo, fue resuelto numéricamente usando la técnica de diferencias finitas explícitas (Ecuación 5). Para esto, se programó un código en MATLAB ${ }^{\circledR}$, que discretizó y aproximó numéricamente esta derivada, permitiendo obtener el gradiente de humedad simulado, para todo el dominio de interés, los que después fueron comparados con las mediciones experimentales.

$\left(\frac{\partial^{2} \mathrm{CH}}{\partial x^{2}}\right)_{i, j}=\frac{\mathrm{CH}_{i, j+1}-\mathrm{CH}_{i, j}+\mathrm{CH}_{i, j-1}}{\left(\Delta x^{2}\right)}$

De modo similar, el coeficiente de difusión efectivo $\left(D_{\mathrm{ef}}\right)$, se obtuvo con modelos propuestos en la literatura (Ecuación 6 y 7), siendo ajustados mediante regresión múltiple no-lineal utilizando el software Curve Expert ${ }^{\circledR}$ 1.4. Los parámetros fueron definidos, mediante un análisis de regresión utilizando el método de los mínimos cuadrados y su nivel de ajuste fue evaluado por su coeficiente de determinación $\left(r^{2}\right)$. Por último, se aplicó una función de error que comparó los coeficientes de difusión generados por el modelo, con las mediciones experimentales (Ecuación 8).

$$
\begin{aligned}
& D_{e f}=a \times \exp \left(\frac{b}{T_{b s}}\right) \times\left(\frac{C H}{100}\right)^{c} \\
& D_{e f}=a \times \exp \left(\frac{b}{T_{b s}}+\left[c \times \frac{C H}{100}\right]\right)
\end{aligned}
$$

Dónde; $\mathrm{D}_{\mathrm{ef}}$ : Coeficiente de difusión efectivo $\left(\mathrm{m}^{2} / \mathrm{s}\right), \mathrm{CH}$ : Contenido de humedad de la madera (\%), Tbs: Temperatura de bulbo seco $\left({ }^{\circ} \mathrm{C}\right)$; a, b y c: Parámetros del modelo.

$$
E C M=\sqrt{\sum_{i=1}^{n} \frac{\left(D_{i}^{\text {mod }}-D_{i}^{e x p}\right)^{2}}{n}} \times 100
$$

Dónde; $E C M$ : Error cuadrático medio en (\%); $D_{i}^{\text {mod: }}$ i-ésimo coeficiente de difusión generado por el modelo; $\mathrm{D}_{\mathrm{i}}^{\mathrm{exp}}$ : i-ésimo coeficiente de difusión experimental; $\mathrm{n}$ : Número de coeficientes de difusión obtenidos por el modelo.

Finalmente, con los coeficientes de difusión simulados, se obtuvo el flujo de humedad para cada instante de tiempo y de esta forma construir la curva de secado simulada. Esta curva, fue ajustada mediante una regresión no lineal, siendo evaluadas por medio de sus coeficientes de determinación $\left(r^{2}\right)$ para cada condición de secado evaluada.

\section{RESULTADOS Y DISCUSIÓN}

Variación del coeficiente de difusión con la temperatura de secado y humedad de la madera.

Los coeficientes de difusión efectivo $\left(D_{\mathrm{ef}}\right)$ obtenidos en este estudio, fueron válidos para temperaturas de bulbo seco ( $\mathrm{T}_{\mathrm{bs}}$ ) entre $40{ }^{\circ} \mathrm{C}-55^{\circ} \mathrm{C}$ y humedad de la madera entre 12,5\%-120\%. Estos coeficientes, fueron ajustados en dos modelos semi-empíricos (Ecuación 9 y 10) derivados de la Ecuación 7 y 8 . Los modelos, fueron similares a los 
desarrollados por Simpson \& Liu (1991, 1997), Simpson (1993), Hukka (1999), Rozas (2007) y Rozas et al. (2009), quienes modelaron al coeficiente de difusión efectivo, por medio de una función de tipo exponencial, dependiente de la humedad y temperatura de secado.

$$
\begin{aligned}
& D_{e f}=5,01126 \times 10^{-9} \exp \left[\frac{-1,25255 \times 10^{2}}{T_{b s}} \times\left(\frac{C H}{100}\right)^{0,1926}\right] \\
& D_{e f}=3,23769 \times 10^{-9} \exp \left[\frac{-1,24461 \times 10^{2}}{T_{b s}}+\left(0,4509 \times \frac{C H}{100}\right)\right]
\end{aligned}
$$

Dónde; $D_{\text {ef: }}$ Coeficiente de difusión efectivo $\left(\mathrm{m}^{2} / \mathrm{s}\right) ; \mathrm{CH}$ : Contenido de humedad de la madera (\%); $\mathrm{T}_{\mathrm{bs}}$ : Temperatura de bulbo seco $\left({ }^{\circ} \mathrm{C}\right)$.

Los coeficientes de difusión efectivo simulados y experimentales, presentaron un orden de magnitud del $10^{-10}\left(\mathrm{~m}^{2} / \mathrm{s}\right)$ (Figura 1). Concordando con Kang et al. (2009), quienes reportaron un orden de magnitud entre $10^{-8}$ y $10^{-10}\left(\mathrm{~m}^{2} / \mathrm{s}\right)$ para la mayoría de especies de interés comercial y donde es esperable que las maderas de secado lento, tengan un orden de magnitud en torno a $10^{-10}\left(\mathrm{~m}^{2} / \mathrm{s}\right)$.

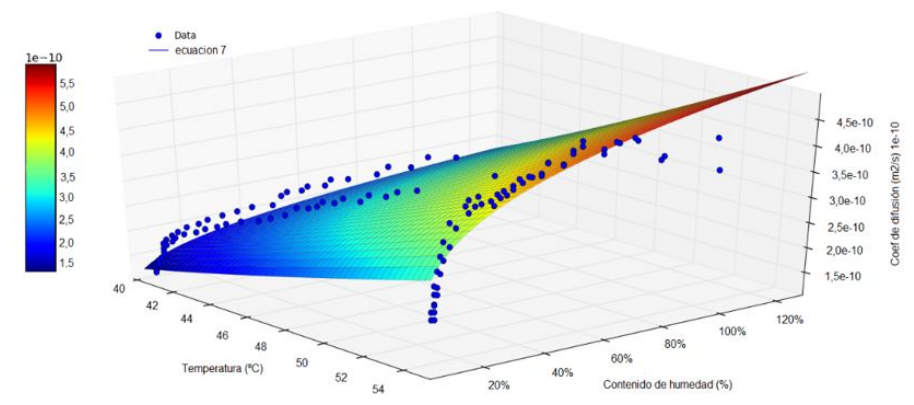

a).

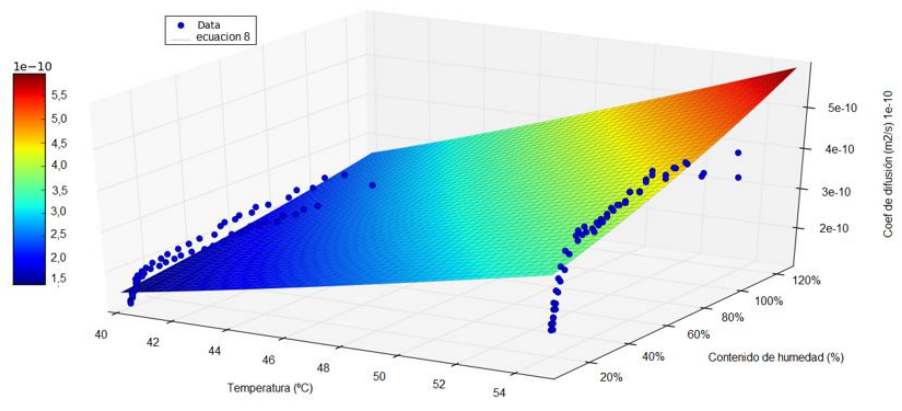

b).

Figura 1. Representación gráfica de los coeficientes de difusión efectivo ( $\left.D_{\mathrm{ef}}\right)$ obtenidos de la Ecuación 9 (a) y Ecuación 10 (b).

De los modelos, se observó una dependencia del coeficiente de difusión efectivo con la temperatura $\left(\mathrm{T}_{\text {bs }}\right)$ y humedad de la madera $(\mathrm{CH})$, comportamiento que también fue reportado por Chen \& Choong (1995), Liu \& Simpson (1999), Liu et al. (2001), Rozas (2007) y Rozas et al. (2009). Además, se observó que el coeficiente de difusión efectivo, presentó valores aproximadamente constantes, cuando la humedad de la madera estuvo entre el contenido de humedad máximo y el punto de saturación de las fibras (PSF). Esto también lo reportaron Chen \& Choong (1995), Hukka (1999), Liu et al. (2001), Rozas (2007) y lo atribuyen a un 
comportamiento de maderas de secado lento y de baja permeabilidad. Lo cual, tiene sentido para el caso del secado de madera de Eucalyptus nitens, debido a que esta especie presenta un flujo del agua capilar altamente restringido, induciendo un transporte de humedad por difusión para la mayor parte del proceso de secado. En este mismo análisis, se detectó una disminución del coeficiente de difusión efectivo, a medida que la humedad de la madera se aproximó al punto de saturación de las fibras (PSF), efecto que se incrementó al momento que la humedad de la madera se aproximó a su humedad de equilibrio (CHE).

De modo similar, hubo un aumento del coeficiente de difusión efectivo con la temperatura del secado, lo cual fue reportado por Hukka (1999), Mukam \& Wanko (2005), Rozas (2007) y Rozas et al. (2009) y quienes lo atribuyen a que el coeficiente de difusión, obedece a la ley de Arrhenius. Con respecto a esto, Biggerstaff (1965), reportó un incremento exponencial del coeficiente de difusión con la temperatura, por lo que debe considerarse a la temperatura como un factor significativo al momento de simular a los coeficientes de difusión efectivo.

La Tabla 2, resume a los coeficientes de determinación $\left(r^{2}\right)$ y error cuadrático medio (ECM) de los modelos semi-empíricos, siendo el modelo descrito por la Ecuación 9, el que presentó el mayor ajuste $\left(r^{2}: 0,9305\right)$ y el menor error (ECM: 0,3023). Mientras que la Ecuación 10, presentó el menor ajuste $\left(r^{2}: 0,8528\right)$ y mayor error $(E C M: 0,4540)$ en comparación a la Ecuación 9.

Tabla 2. Coeficiente de determinación $\left(r^{2}\right)$ y error cuadrático medio $(E C M)$ para los coeficientes de difusión efectivo $\left(D_{\mathrm{ef}}\right)$ descritos por la ecuación 9 y ecuación 10.

\begin{tabular}{ccc}
\hline & Ecuación $\mathbf{9}$ & Ecuación $\mathbf{1 0}$ \\
\hline$r^{2}$ & 0,9305 & 0,8528 \\
$E C M$ & 0,3023 & 0,4540 \\
\hline
\end{tabular}

\section{Modelación del gradiente de humedad.}

Los gradientes de humedad experimentales y simulados se muestran en la Figura 2 $\left(40 / 35^{\circ} \mathrm{C}\right.$ ) y Figura $3\left(55 / 50^{\circ} \mathrm{C}\right.$ ), observando para las primeras 192 horas (condición $40 / 35^{\circ} \mathrm{C}$ ) y las primeras 114 horas (condición $55 / 50{ }^{\circ} \mathrm{C}$ ), valores experimentales mayores a los simulados. Para Rozas (2007), la diferencia entre los valores simulados y experimentales, se debe a variaciones en las condiciones psicrométricas al interior de la cámara de secado. Sin embargo, a medida que el secado avanzó y la madera se aproximó al punto de saturación de las fibras (PSF), el modelo reprodujo con mayor precisión al gradiente de humedad, lo cual se mantuvo hasta alcanzar la humedad de equilibrio de la madera (CHE). Este comportamiento también fue reportado por Hart \& Darwin (1971) y Plumb et al. (1985), quienes indicaron que el modelo difusivo, subestima al gradiente de humedad en las primeras horas del secado, pero a medida el secado avanza, se incrementa su precisión, siendo esto también observado en especies de baja permeabilidad. 

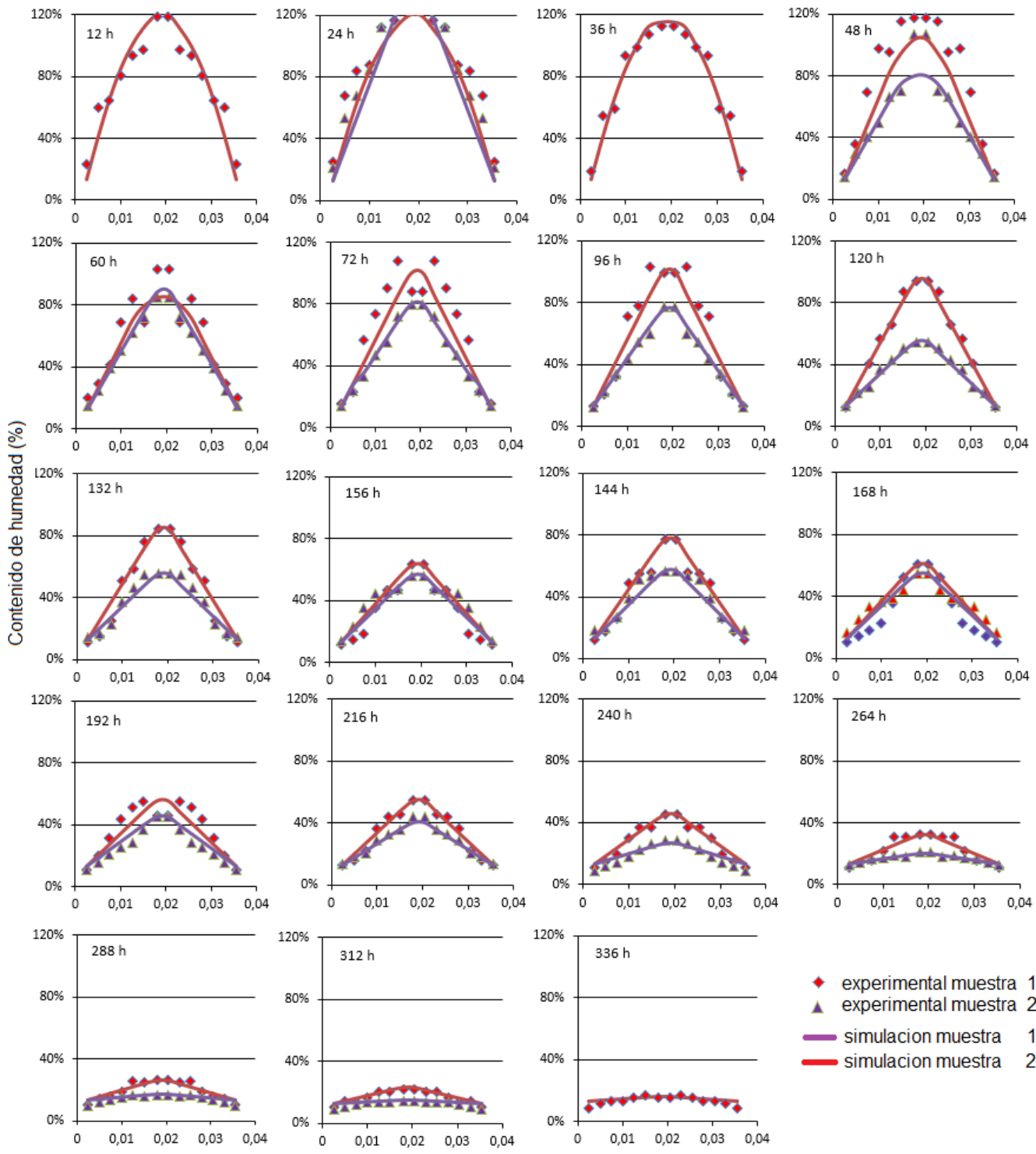

- experimental muestra 1

experimental muestra 2

simulacion muestra 1

simulacion muestra 2

Espesor (m)

Figura 2. Gradiente de humedad simulado (línea) y experimental (puntos) para secado isotérmico a condición $40 / 35^{\circ} \mathrm{C}$ en madera de Eucalyptus nitens. 

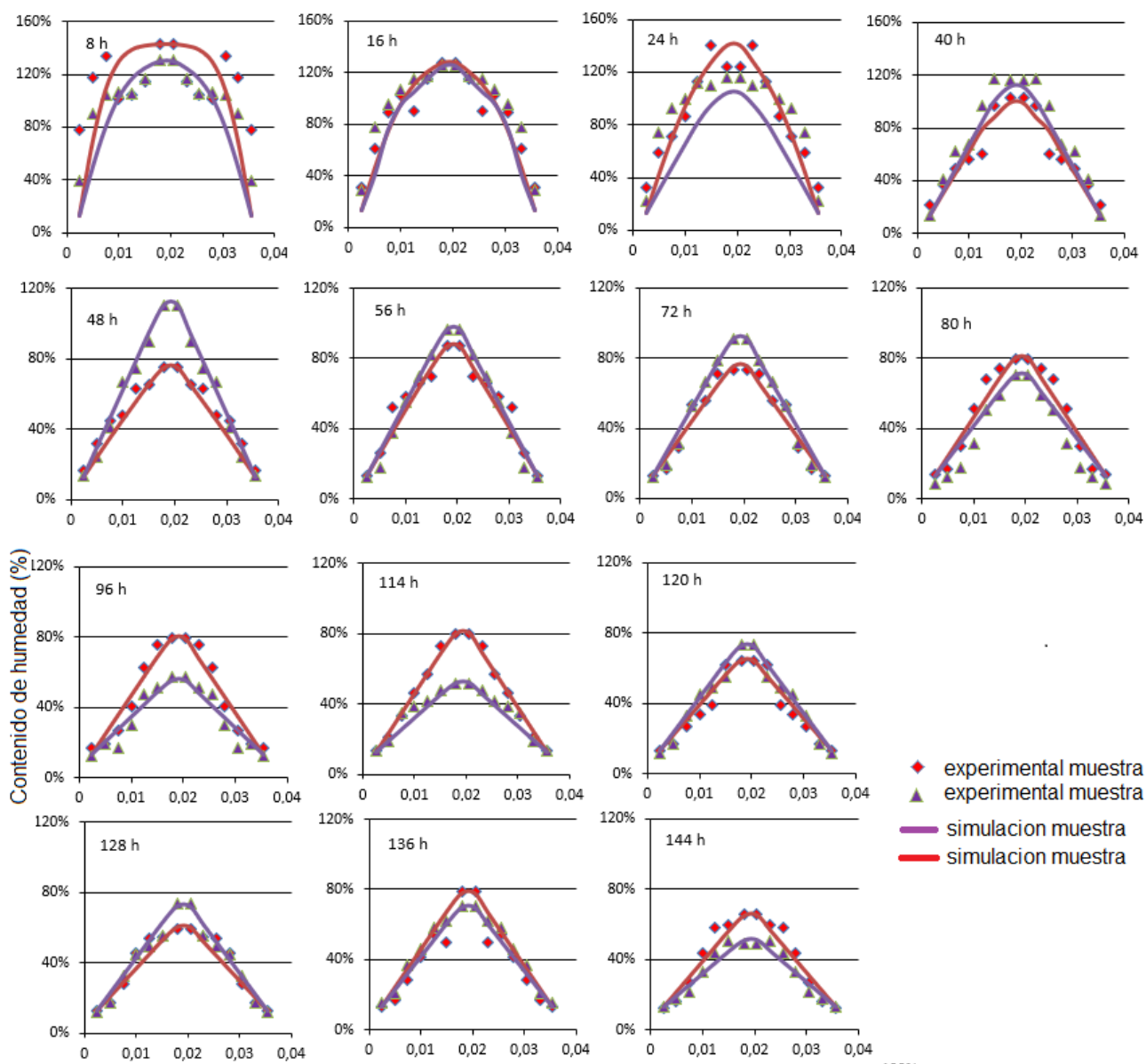

experimental muestra

- experimental muestra 2

- simulacion muestra 1

simulacion muestra 2
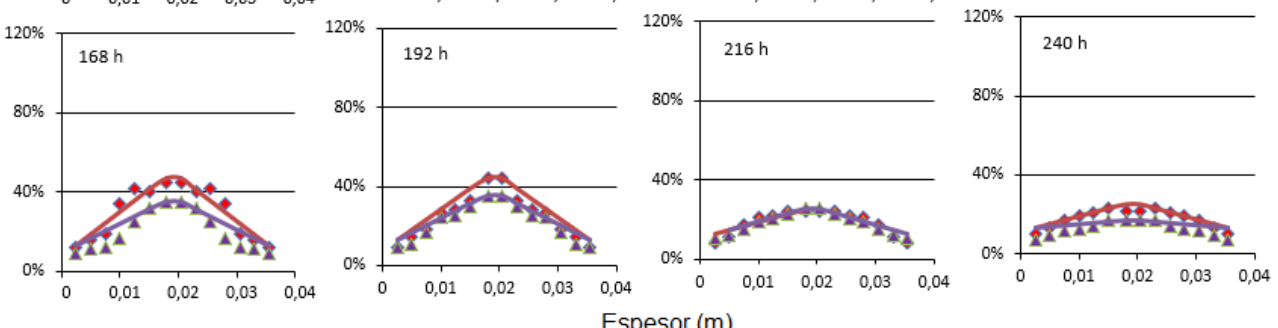

Figura 3. Gradiente de humedad simulado (línea) y experimental (puntos) para secado isotérmico a condición de $55 / 50{ }^{\circ} \mathrm{C}$ en madera de Eucalyptus nitens.

Con respecto a la humedad superficial de la madera, esta alcanzó el punto de saturación de las fibras alrededor de las $12 \mathrm{~h}$, este dato debe considerarse relevante ya que es un instante critico durante el proceso de secado. Ya que, bajo estas condiciones superficiales, se da comienzo de las tensiones de secado superficiales, las que al incrementarse ocasionan grietas superficiales que reducen la calidad final de la madera (Rozas, 2007). Mientras que para Kirk et al. (1985), cuando la madera alcanza el PSF en su superficie, a un tiempo que en proporción es bajo con respecto al ciclo de secado, queda en evidencia que el transporte de humedad, ocurre principalmente por difusión.

\section{Modelación de la cinética de secado.}

La Figura 4 y 5 muestra las curvas de secado experimental y simuladas para la condición $40 / 35{ }^{\circ} \mathrm{C}$ y $55 / 50{ }^{\circ} \mathrm{C}$ respectivamente. Las curvas simuladas, presentaron una adecuada precisión para ambas condiciones de secado. Sin embargo, se detectó durante las primeras horas del secado, que el modelo subestimó el valor real de la humedad en la madera. Esto también fue reportado por Hart \& Darwin (1971) y Rozas (2007) y lo atribuyen al secado de 
maderas de baja permeabilidad y con flujo de agua capilar altamente restringido, los cuales reducen la transferencia de masa y hace imprecisa la simulación al comienzo del secado. Sin embargo, a medida que el secado avanzó, se incrementó la precisión de la curva de secado simulada, lo cual ocurrió aproximadamente a las 36 horas para ambas condiciones de secado. En general, la simulación de la curva de secado y del gradiente de humedad, se puede considerar como adecuada para las condiciones de secado evaluadas. Por lo que, en vista de los resultados obtenidos, se puede emplear este tipo de modelos para la predicción del secado en madera aserrada de Eucalyptus nitens.
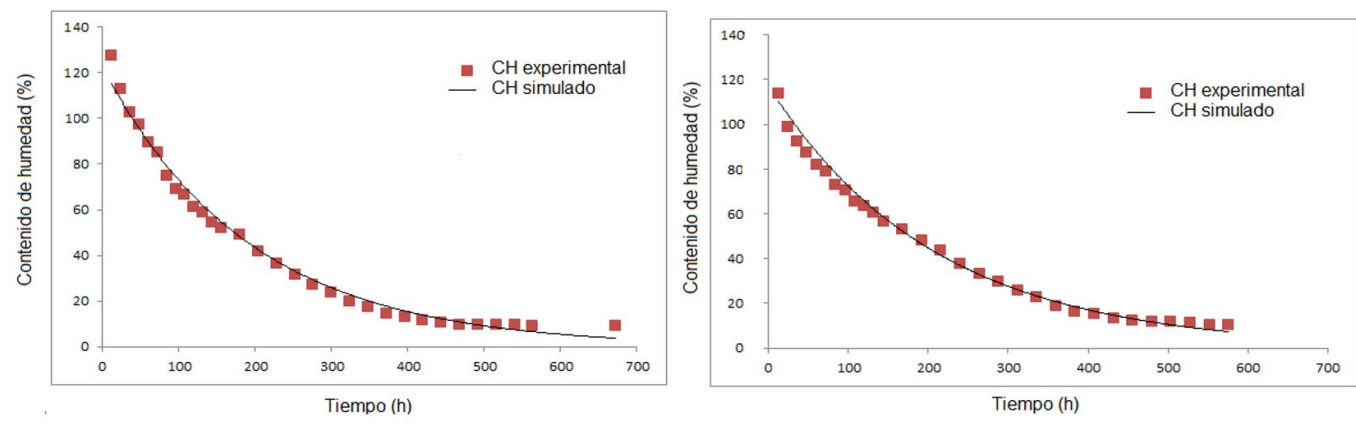

Figura 4. Curva de secado simulada (líneas) y experimental (cuadrados) para la condición de secado de $40 / 35^{\circ} \mathrm{C}$.
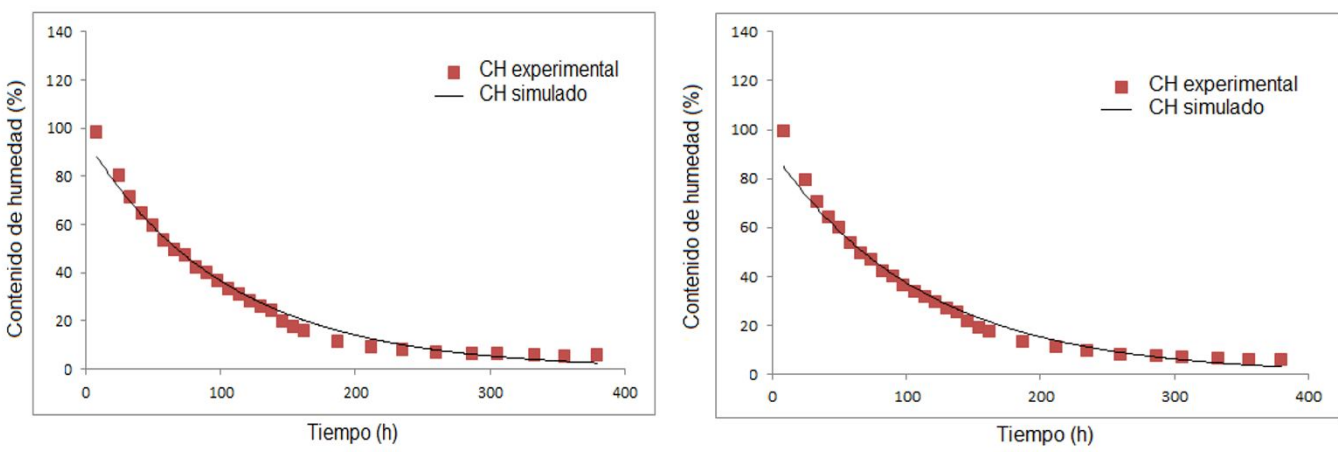

Figura 5. Curva de secado simulada (líneas) y experimental (cuadrados) para la condición de secado de $55 / 50^{\circ} \mathrm{C}$.

\section{CONCLUSIONES}

De los resultados obtenidos en esta investigación se concluyó que:

El gradiente de humedad y la curva de secado simulados en madera de Eucalyptus nitens, presentaron alta precisión, la cual se incrementó a medida que la humedad de la madera se aproximó al contenido de humedad de equilibrio.

Se demostró que el modelo difusivo, puede emplearse para identificar las diferentes etapas de secado, tal como es el caso donde se producen tensiones de secado y grietas superficiales, haciedolo útil para definir estrategias de secado y así incrementar el valor y la calidad final del secado de esta especie.

Esta metodología puede ser replicada en otras especies y condiciones de secado, con el fin de identificar opciones de mejora del proceso, presentando como principal ventaja, su reducida cantidad de parámetros y su bajo costo computacional.

\section{RECOMENDACIONES.}

Se recomienda realizar ensayos que evalúen otras condiciones de secado y escuadrías de la madera, no exploradas en esta investigación. Lo anterior, para ampliar el conocimiento tecnológico del secado y de esta_forma incrementar el rendimiento, calidad y valor agregado de la madera de Eucalyptus nitens 


\section{REFERENCIAS}

Avramidis, S., \& Siau, J. (1987). An investigation of the external and internal resistance to moisture diffusion in wood. Wood Science and Technology, 21(3), 249-256.

Baronas, R., Ivanauskas, F., \& Sapagovas, M. (2002). Reliability of one-dimensional model of moisture diffusion in wood. Informatica, 13(4), 405-416.

Biggerstaff, T. (1965). Drying diffusion coefficients in wood as affected by temperature. Forest Products Journal, 25(3), 127-133.

Blakemore, P. A., \& Langrish, T. A. G. (2008). Effect of collapse on fitted diffusion coefficients for Victorian ash Eucalypts. Wood Science and Technology, 42(7), 535-549. http://dx.doi.org/10.1007/s00226-008-0184-7.

Bramhall, G. (1979). Mathematical model for lumber drying. Part I principles involved. Wood Science, 12(1), 22-30.

Bui, X., Chong, E., \& Rudd, W. (1980). Numerical methods for solving the equation for diffusion through wood during drying. Wood Science, 13(2), 117-121.

Cai, L. (2005). Determination of diffusion coefficients for sub-alpine fir. Wood Science and Technology, 39(2), 153-162. http://dx.doi.org/10.1007/s00226-004-0284-y.

Chen, Y., \& Choong, E. T. (1995). Evaluation of diffusion coefficient and surface emission coefficient by an optimization technique. Wood and Fiber Science, 27(2), 178-182.

Chen, Y., Choong, E. T., \& Wetzel, D. M. (1996). A numerical analysis technique to evaluate the moisturedependent diffusion coefficient on moisture movement during drying. Wood and Fiber Science, 28(3), 338-345.

Dirección Meteorológica de Chile. (2001). Climatología regional (pp. 45). Santiago de Chile: Dirección Meteorológica de Chile. Recuperado el 13 de julio de 2018, de http://164.77.222.61/climatologia/

Eriksson, J., Johansson, H., \& Danvind, J. (2007). A mass transport model for drying wood under isothermal conditions. Drying Technology, 25(3), 433-467. http://dx.doi.org/10.1080/07373930601183785.

Gatica, Y., Salinas, C., \& Ananias, R. (2011). Modeling conventional one-dimensional drying of radiata pine based on the effective diffusion coefficient. Latin American Applied Research, 41(2), 183-189.

Hart, C. A. (1964). Principles of moisture movement in wood. Forest Products Journal, 14(5), 207-214.

Hart, C. A., \& Darwin, W. M. (1971). The slow drying rate of white oak. Wood Science, 4(1), 46-54.

Hukka, A. (1999). The effective diffusion coefficient and mass transfer coefficient of Nordic softwoods as calculated from direct drying experiments. Holzforschung, 53(5), 534-540. http://dx.doi.org/10.1515/HF.1999.088.

Instituto Nacional de Normalizacion - INN. (1984). NCh 176/1. Of 1984. Madera Parte 1: Determinación de humedad (7 p.). Santiago de Chile.

Kang, W., Lee, Y. H., Chung, W. Y., \& Xu, H. L. (2009). Parameter estimation of moisture diffusivity in wood by an inverse method. Journal of Wood Science, 55(2), 83-90. http://dx.doi.org/10.1007/s10086008-1006-0.

Kirk, C., Choong, T., \& Wetzel, D. (1985). Mathematical modeling of the diffusion of water in wood during drying. Wood and Fiber Science, 17(3), 404-423.

Li, X., Zhang, B., Li, W., \& Li, Y. (2005). Research on the effect of microwave pretreatment on moisture diffusion coefficient of wood. Wood Science and Technology, 39(7), 521-528. http://dx.doi.org/10.1007/s00226-005-0007-z.

Liu, J. Y. (1989). A new method for separating diffusion coefficient and surface emission coefficient. Wood and Fiber Science, 21(2), 133-141.

Liu, J. Y., \& Simpson, W. (1999). Two-stage moisture diffusion in wood with constant transport coefficients. Drying Technology, 17(2), 257-269. http://dx.doi.org/10.1080/07373939908917528.

Liu, J. Y., Simpson, W., \& Verrill, S. (2001). An inverse moisture diffusion algorithm for the determination of diffusion coefficient. Drying Technology, 19(8), 1555-1568. http://dx.doi.org/10.1081/DRT100107259.

Mukam, J. A., \& Wanko, C. (2005). Experimental determination of the diffusion coefficients of wood in isothermal conditions. Heat and Mass Transfer, 41(11), 977-980. http://dx.doi.org/10.1007/s00231005-0621-1. 
Nadler, K. C., Choong, E. T., \& Wetzel, D. M. (1985). Mathematical modeling of the diffusion of water in wood during drying. Wood and Fiber Science, 17(3), 404-423.

Olek, W., \& Weres, J. (2007). Effects of the method of identification of the diffusion coefficient on accuracy of modeling bound water transfer in wood. Transport in Porous Media, 66(2), 135-144. http://dx.doi.org/10.1007/s11242-006-9010-6.

Pang, S. (1996). Moisture content gradient in a softwood board during drying: simulation from 2-D model and measurement. Wood Science and Technology, 30(3), 165-178. http://dx.doi.org/10.1007/BF00231631.

Plumb, O., Spolek, G., \& Olmstead, B. A. (1985). Heat and mass transfer in wood during drying. International Journal of Heat and Mass Transfer, 28(9), 1669-1678. http://dx.doi.org/10.1016/00179310(85)90141-3.

Resch, H., Kang, H., \& Hoag, M. L. (1989). Drying Douglas-Fir lumber: a computer simulation. Wood and Fiber Science, 21(3), 207-218.

Rozas, C. (2007). Modelo de transferência de calor e massa na secagem de madeira serrada de pinus (Tese de doutorado). Universidade Federal do Paraná, Curitiba.

Rozas, C., Tomaselli, I., \& Zanoelo, E. F. (2009). Internal mass transfer coefficient during drying of softwood (Pinus elliottii Engelm.) boards. Wood Science and Technology, 45(5), 361-373. http://dx.doi.org/10.1007/s00226-008-0223-4.

Simpson, W. T. (1993). Determination and use of moisture diffusion coefficient to characterize drying of northern red oak (Quercus rubra). Wood Science and Technology, 27(6), 409-420. http://dx.doi.org/10.1007/BF00193863.

Simpson, W. T., \& Liu, J. Y. (1991). Dependence of the water diffusion coefficient of aspen (Populus spec.) on moisture content. Wood Science and Technology, 29(1), 9-21. http://dx.doi.org/10.1007/BF00225688.

Simpson, W., \& Liu, J. Y. (1997). An optimization technique to determine red oak surface and internal moisture transfer coefficients during drying. Wood and Fiber Science, 29(4), 312-318.

Author's contributions: CM: Conservación de datos, análisis formal, investigación, metodología, administración del proyecto, software, visualización, redacción-borrador original, redacción-revisión \& edición;

CR: Conceptualización, análisis formal, adquisición de fondos, metodología, recursos, supervisión, validación, redacción-borrador original, redacción-revisión \& edición; CP: análisis formal, software, recursos. 